\title{
Cobertura de plano de saúde no Brasil: análise dos dados da Pesquisa Nacional de Saúde 2013 e 2019
}

\author{
Health insurance coverage in Brazil: \\ analyzing data from the National Health Survey, 2013 and 2019
}

Paulo Roberto Borges de Souza Júnior (https://orcid.org/0000-0002-8142-4790) ${ }^{1}$
Célia Landmann Szwarcwald (https://orcid.org/0000-0002-7798-2095) ${ }^{1}$
Giseli Nogueira Damacena (https://orcid.org/0000-0002-7059-3353) ${ }^{1}$
Sheila Rizzato Stopa (https://orcid.org/0000-0001-8847-665X) ${ }^{2}$
Maria Lúcia França Pontes Vieira (https://orcid.org/0000-0002-5284-6214) ${ }^{3}$
Wanessa da Silva de Almeida (https://orcid.org/0000-0002-5164-8603) ${ }^{1}$
Max Moura de Oliveira (https://orcid.org/0000-0002-0804-5145) ${ }^{2}$
Luciana Monteiro Vasconcelos Sardinha (https://orcid.org/0000-0002-3679-3618) ${ }^{2}$
Eduardo Marques Macário (https://orcid.org/0000-0002-6383-0365) ${ }^{2}$

${ }^{1}$ Instituto de Comunicação e Informação Científica e Tecnológica em Saúde, Fundação Oswaldo Cruz. Av. Brasil 4635, Manguinhos, Pavilhão Haity Moussatché. 21045-360 Rio de Janeiro RJ Brasil. paulo.borges@icict.fiocruz.br ${ }^{2}$ Departamento de Análise em Saúde e Vigilância de Doenças Não Transmissíveis, Secretaria de Vigilância em Saúde, Ministério da Saúde. Brasília DF Brasil.

${ }^{3}$ Diretoria de Pesquisas, Instituto Brasileiro de

Geografia e Estatística. Rio de Janeiro RJ Brasil.

\begin{abstract}
This paper aimed to describe health insurance coverage in Brazil. Data from the 2013 and 2019 editions of the National Health Survey (PNS) were analyzed. The medical or dental health insurance coverage was analyzed according to demographic and socioeconomic characteristics, work status, urban/rural area, and Federation Unit. Coverage of medical or dental health insurance was $27.9 \%$ (95\% CI: 27.1-28.8) for 2013 and 28.5\% (95\% CI: 27.829.2) for 2019. The results show coverage is still concentrated in large urban centers, in the Southeast and South, among those with better socioeconomic status and some formal employment. In 2019, only 30.7\% of formal workers reported the monthly payment is made directly to the providers, while $72.7 \%$ of informal workers reported this information. About $92 \%$ of medical health insurance covers hospitalization, and almost 20\% of women with health insurance are not covered for labor. Only $11.7 \%$ of women aged between 15 and 44 are covered for childbirth by health insurance. The results show the health insurance coverage is still quite unequal, reinforcing the Unified Health System (SUS) importance for the Brazilian population.

Key words Prevalence of private health insurance plan, Prepaid health insurance plan, Epidemiological surveys, Brazil
\end{abstract}

Resumo Este artigo objetivou descrever a cobertura de plano de saúde no Brasil. Foram analisados dados das edições de 2013 e 2019 da Pesquisa Nacional de Saúde. A cobertura de plano de saúde médico ou odontológico foi analisada segundo características sociodemográficas, econômicas, de trabalho, situação censitária e Unidade da Federação. A cobertura de plano de saúde médico ou odontológico foi 27,9\% (IC95\%: 27,1-28,8) para 2013 e 28,5\% (IC95\%: 27,8-29,2) para 2019. Os resultados mostram que a cobertura continua concentrada nos grandes centros urbanos, nas regiões Sudeste e Sul, entre aqueles com melhor nível socioeconômico e aqueles que possuem algum vinculo de trabalho formal. Em 2019, dentre os trabalhadores formalizados, somente 30,7\% relatou que o pagamento da mensalidade é feito diretamente a operadora, sendo $72,7 \%$ dentre os trabalhadores informais. Cerca de $92 \%$ dos planos de saúde médico cobrem internação e dentre as mulheres com plano de saúde, quase $20 \%$ delas não possuem cobertura para o parto. Apenas $11,7 \%$ das mulheres com idade entre 15 e 44 anos possuem cobertura para o parto através do plano de saúde. Os resultados mostram que a cobertura por plano de saúde mantém-se bastante desigual, reforçando a importância do Sistema Único de Saúde para a população brasileira.

Palavras-chave Cobertura de planos de saúde, Plano de saúde pré-pago, Inquéritos epidemiológicos, Brasil 


\section{Introdução}

O sistema de saúde brasileiro tem como característica a coexistência de serviços públicos e privados, tanto em relação a estrutura física quanto ao financiamento. De um lado, o Sistema Único de Saúde (SUS), pautado nos princípios de universalidade, integralidade e equidade, com abrangência nacional e ampla cobertura territorial, oferece serviços gratuitos a toda população em todos os níveis de atenção. De outro, o setor privado, idealizado e normatizado pela Constituição Federal de $1988^{1}$ para ser organizado de forma complementar aos serviços públicos prestados pelo SUS, oferecendo serviços não cobertos pelo setor público. Porém, na realidade, existe uma sobreposição de serviços, onde o setor privado oferta serviços que já são disponibilizados de forma gratuita no setor público. Sendo assim, o mercado de planos e seguros privados atua de forma suplementar, gerando uma cobertura duplicada, onde seus segurados podem acessar tanto os serviços públicos quanto os privados ${ }^{2,3}$.

Santos et al. ${ }^{2}$ ressaltam que este tipo de cobertura pode gerar alguns problemas tanto em relação ao acesso aos serviços de saúde, quanto aos gastos públicos, pois além de continuarem a usar os serviços públicos, os gastos com o pagamento do plano de saúde são deduzidos do imposto de renda devido, transferindo para o governo os gastos com o setor privado de saúde.

Os planos de saúde existem no Brasil desde $1940^{4} \mathrm{e}$ no ano 2000 foi criada a Agência Nacional de Saúde Suplementar (ANS), pela Lei Federal no $9.961^{5,6}$ com o intuito de regular o setor, elaborando normas e fiscalizando as operadoras dos planos de saúde.

Em 1998, por meio do suplemento de saúde da Pesquisa Nacional por Amostra de Domicílios (PNAD) do Instituto Brasileiro de Geografia e Estatística (IBGE), estimou-se que $24,5 \%$ da população brasileira era coberta por algum plano de saúde. Esta proporção se manteve semelhante até 2003, quando foi estimada uma cobertura de 24,6\% (dados obtidos na página de informações em saúde do Tabnet do DATASUS, no submenu de inquéritos e pesquisas: http://tabnet.datasus. gov.br/cgi/menu_tabnet_php.htm\#).

No ano de 2013, o IBGE em conjunto com o Ministério da Saúde (MS) e a Fundação Oswaldo Cruz (Fiocruz), foi a campo com a Pesquisa Nacional de Saúde (PNS), com o objetivo de produzir dados em âmbito nacional sobre a situação de saúde e os estilos de vida da população brasilei$\mathrm{ra}^{7,8}$. Por meio da PNS, que substituiu o suplemen- to de saúde da PNAD, foi estimada uma cobertura de $27,8 \%$ de plano de saúde para a população brasileira. A PNS foi realizada novamente em 2019.

A importância do monitoramento da cobertura de plano de saúde se dá tanto para a regulação do setor quanto para os gestores de saúde, no planejamento do setor público de saúde, no que diz respeito à oferta de serviços e ao tamanho da população coberta somente pelo SUS.

Este artigo tem o objetivo de descrever a cobertura de plano de saúde, estimada para os anos de 2013 e 2019, por meio das duas edições da PNS, estratificando por características sociodemográficas, econômicas e de trabalho, avaliando, quando possível as diferenças nas coberturas médico-hospitalar e odontológica.

\section{Métodos}

Este estudo analisou dados da Pesquisa Nacional de Saúde realizada em duas edições, nos anos de 2013 e 2019. A Pesquisa Nacional de Saúde de 2019 (PNS/2019) é um inquérito domiciliar realizado pelo Instituto Brasileiro de Geografia e Estatística (IBGE) em parceria com o Ministério da Saúde (MS) cujo objetivo é produzir dados sobre a situação de saúde e os estilos de vida da população brasileira. O questionário da $\mathrm{PNS} / 2019$ foi dividido em três partes: (1) informações do domicílio, (2) informações de todos os moradores e (3) informações sobre um indivíduo com 15 anos ou mais selecionado aleatoriamente. A população alvo foi composta pelas pessoas residentes em domicílios particulares permanentes (DPP) em todo o território nacional.

A PNS/2019 faz parte do Sistema Integrado de Pesquisas Domiciliares (SIPD) que tem como estrutura amostral a Amostra Mestra. A Amostra Mestra é um conjunto de setores censitários ou agregados de setores selecionados para atender a diversas pesquisas realizadas pelo IBGE. Estes setores formam as unidades primárias de amostras das pesquisas e são estratificados segundo quatro critérios: Administrativo (Unidades da Federação - UF, capitais, Regiões Metropolitanas, Região Integrada de Desenvolvimento Econômico - RIDE e demais setores da UF); Geográfico (Subdivisões das capitais e outros municípios de grande porte em áreas como distritos, subdistritos e bairros); De Situação (urbano e rural) e um critério estatístico, que subdivide os estratos baseados nos três critérios anteriores em estratos homogêneos, segundo as informações de rendimento total dos domicílios e número de domicílios particulares ${ }^{8}$. 
A amostra da PNS/2019 é probabilística com amostragem por conglomerado em três estágios de seleção. No primeiro estágio, foram selecionadas 8.036 unidades primárias de amostragem (UPA) dentro do quantitativo de UPA da Amostra Mestre, respeitando a estratificação da mesma. As UPA correspondem a setores censitários ou conjunto de setores. No segundo estágio, foram selecionados de 12 a 18 domicílios em cada UPA, totalizando 108.525 domicílios e no terceiro estágio foi selecionado aleatoriamente, dentro de cada domicílio, um indivíduo de 15 anos ou mais para responder a terceira parte do questionário, referente a pessoa selecionada. A amostra final corresponde a 94.114 domicílios com entrevista realizada, com uma taxa de resposta de 93,6\%. Os fatores de expansão correspondem ao inverso do produto das probabilidades de seleção em cada estágio, incluindo um fator de correção para as perdas. Os fatores de expansão foram calibrados levando em consideração as projeções populacionais para o Brasil e Unidades da Federação?.

A Pesquisa Nacional de Saúde 2013 (PNS/2013) possui desenho amostral semelhante, com uma amostra de 64.348 domicílios. Maiores detalhes podem ser obtidos em SouzaJunior et al. ${ }^{8}$. Neste estudo foram utilizados dados referentes a todos os moradores dos domicílios selecionados, de ambas as edições da PNS.

Para permitir comparações entre as edições 2013 e 2019 da PNS, o IBGE realizou uma nova calibração dos fatores de expansão da PNS/2013 considerando a revisão da Projeção da População das Unidades da Federação por Sexo e Idade, para o período de 2010-2060, divulgada em 2018. Esta mesma projeção populacional foi utilizada na calibração dos pesos da PNS/2019, garantindo assim a comparabilidade entre as duas edições da pesquisa.

A PNS/2019 possui duas perguntas sobre a posse de plano de saúde, "tem algum plano odontológico particular, de empresa ou órgão público?" e "tem algum plano de saúde médico particular, de empresa ou órgão público?”. Porém, na PNS/2013, uma única questão englobava os dois tipos de cobertura, "tem algum plano de saúde, médico ou odontológico, particular, de empresa ou órgão público?”. Embora haja uma outra questão sobre posse de plano de saúde exclusivamente odontológico, "tem algum plano de saúde apenas para assistência odontológica?”, ela não permite separar aqueles com cobertura somente médica. Por este motivo, optou-se por avaliar conjuntamente a cobertura médica e/ou odontológica nas comparações entre 2013 e 2019.
A cobertura de plano de saúde médico ou odontológico foi analisada segundo as variáveis sexo (masculino e feminino); faixa etária $(<18$, 18-29, 30-59 e 60 anos ou mais); cor ou raça (branca, preta, parda e outra); nível de escolaridade (Fundamental incompleto ou equivalente incluindo sem instrução, Fundamental completo ou equivalente, Médio completo ou equivalente $\mathrm{e}$ Superior completo ou mais); faixa de rendimento domiciliar per capita, em salários mínimos (SM) (até $1 / 4$ SM, mais de $1 / 4$ até $1 / 2 \mathrm{SM}$, mais de $1 / 2$ até $1 \mathrm{SM}$, mais de 1 até $2 \mathrm{SM}$, mais de 2 até 3 $\mathrm{SM}$, mais de 3 até $5 \mathrm{SM}$ e mais de $5 \mathrm{SM}$ ); condição em relação à força de trabalho (pessoas na força de trabalho, pessoas fora da força de trabalho); e condição de ocupação (pessoas ocupadas, pessoas desocupadas). As questões sobre trabalho da PNS/2019 permitem diferenciar, dentre os ocupados, aqueles que possuem um emprego formal, como os servidores públicos e os empregados com carteira assinada. Sendo assim, para 2019 foi criada uma variável denominada "Situação em relação a ocupação e força de trabalho", com as seguintes categorias: ocupado em emprego formal (servidor público, trabalhador com carteira assinada, empregador ou militar), ocupado sem carteira assinada, desocupado e fora da força de trabalho.

A posse de plano de saúde também foi analisada segundo a Unidade da Federação (UF), Grande Região (Norte, Nordeste, Sudeste, Sul e Centro-Oeste), Situação censitária (urbano ou rural) e tipo de área: capital, Região Metropolitana, RIDE (Região Integrada de Desenvolvimento) e interior (resto da UF).

Para aqueles que possuem plano de saúde médico, foi possível analisar o nível de satisfação com o plano de saúde através da seguinte pe gunta, "considera este plano de saúde:", com as seguintes opções de resposta: muito bom, bom, regular, ruim e muito ruim. Neste estudo foi calculado o percentual de pessoas que consideravam o plano de saúde como bom ou muito bom. Este indicador foi calculado somente para 2019, pois em 2013 esta pergunta foi feita para todos os indivíduos que possuíam plano de saúde médico ou odontológico e em 2019 somente aqueles que possuíam plano de saúde médico puderam respondê-la, dificultando a comparação.

Para 2019, foi possível avaliar se o plano de saúde médico cobre internações e parto e o percentual destas coberturas foi analisado segundo as variáveis já descritas anteriormente. Porém, a renda per capita foi recodificada para ter apenas quatro categorias (até $1 / 2 \mathrm{SM}$, mais de $1 / 2$ até $1 \mathrm{SM}$, 
mais de 1 até 2 SM e mais de 2 SM), para aumentar a precisão das estimativas, tendo em vista que estas análises se limitaram aquelas pessoas com posse de plano de saúde.

Para a população coberta por plano de saúde médico na PNS/2019, também foi analisada a pergunta "Quem paga a mensalidade deste plano de saúde?", considerando as seguintes opções de resposta: somente o empregador (atual ou anterior), parte o titular e parte o empregador (atual ou anterior) e somente o titular, diretamente ao plano. Estas três opções representam 95\% do total de respostas para esta questão. As demais opções eram: somente outro morador do domicílio, pessoa não moradora do domicílio e outro e foram agregadas em uma opção denominada "Outros". A análise de quem paga a mensalidade do plano de saúde médico foi feita segundo a situação em relação a ocupação e força de trabalho (descrita anteriormente).

Por fim, para avaliar diferenças no tipo de cobertura segundo as faixas de rendimento domiciliar per capita e a situação em relação a ocupação e força de trabalho, para 2019, foi criada uma variável com o tipo de plano de saúde, discriminando se o plano era exclusivamente médico, se era simultaneamente médico e odontológico ou se era exclusivamente odontológico.

As estimativas foram obtidas levando-se em consideração o desenho amostral das duas pesquisas, incluindo os fatores de expansão e os efeitos de conglomeração. Os dados foram analisados utilizando o pacote estatístico IBM SPSS Statistics - versão $21^{10}$ através do módulo de amostras complexas (Complex Sample). Foram calculados os percentuais e seus respectivos intervalos de confiança (95\%). Para verificar a associação significativa entre variáveis categóricas foi utilizado o teste de F ajustado de Wald.

Para testar diferenças entre as proporções estimados para 2013 e 2019, foi utilizado o teste $t$ de Student para amostras independentes, tendo em vista que as amostras nas duas edições da pesquisa foram selecionadas independentemente. As proporções estimadas e suas respectivas variâncias foram calculadas considerando o desenho complexo das amostras ${ }^{11}$ e, devido as múltiplas comparações realizadas e ao tamanho da amostra nas duas pesquisas, optou-se por considerar significativas apenas as diferenças cujo valor de $\mathrm{p}$ foi menor que $0,01^{12}$.

\section{Resultados}

Em 2019, a cobertura de plano de saúde médico ou odontológico foi estimada em 28,5\% (IC95\%: 27,8\%-29,2\%), representando uma população de 59,7 milhões de pessoas, levemente superior a estimativa de 55,7 milhões de pessoas cobertas em 2013 (27,9\%; IC95\%: 27,1\%-28,8\%), embora esta diferença não tenha sido significativa. A cobertura de plano de saúde é concentrada nas áreas urbanas (37,7\% em 2013 e 32,2\% em 2019), nas capitais (40,1\% em 2013 e 42,4\% em 2019) e nas regiões Sudeste (37,5\% em 2019) e Sul $(32,8 \%$ em ambos os períodos). Houve um forte gradiente em relação à escolaridade e à renda per capita, sendo maior a cobertura quanto maior a escolaridade e a renda, chegando a $88 \%$ (em 2019) entre aqueles com renda per capita maior que cinco SM (Tabela 1). A cobertura também foi maior entra as pessoas ocupadas, chegando a 47,4\% (em 2019) entre aqueles com empregos considerados formais por este estudo, ou seja, servidores públicos, militares, empregadores e empregados com carteira de trabalho assinada (Tabela 1).

A Figura 1 apresenta as coberturas de plano de saúde médico ou odontológico por Unidade da Federação (UF) para os anos de 2013 e 2019. As maiores coberturas foram nas UF de São Paulo, Rio de Janeiro e Rio Grande do Sul, além do Distrito Federal. Apesar da cobertura ter aumentado em algumas UF e ter diminuído em outras, as diferenças não foram significativas, com exceção do Piauí, cuja cobertura passou de 11,8\% (IC95\%: 10,2\%-13,6\%) para 16,7\% (IC95\%: $14,3 \%-19,4 \%)$ e do Rio de Janeiro, que passou de 32,5\% (IC95\%: 30,3\%-34,9\%) para 37,7\% (IC95\%: 35,7\%-39,8\%). O Mato Grosso e o Mato Grosso do Sul apresentaram as maiores reduções nas estimativas pontuais de cobertura, porém, com valores de p maiores que 0,01 (0,021 e 0,011, respectivamente).

A proporção de pessoas que consideram o plano de saúde como bom ou muito bom foi $79,2 \%$ (IC95\%: 78,3\%-80,0\%) para o Brasil, considerando somente o ano de 2019 . Os residentes na Região Nordeste do país foram aqueles que avaliaram em menor proporção o plano de saúde como bom ou muito bom (73,8\%), enquanto na Região Sul o percentual chegou a $82,4 \%$ (Tabela 2).

Entre aqueles que possuem plano de saúde médico, segundo dados da PNS/2019, em 91,6\% dos casos, o plano cobre internação, com este percentual variando de $80 \%$ entre aqueles com renda per capita até um quarto do SM, e 96,6\% entre aqueles que possuem nível superior de instrução. 
Tabela 1. Percentual de pessoas com posse de plano de saúde médico ou odontológico e intervalo de confiança de 95\%, segundo características sociodemográficas. Brasil, 2013 e 2019.

\begin{tabular}{|c|c|c|c|c|}
\hline \multirow{2}{*}{ Variáveis } & \multicolumn{2}{|r|}{2013} & \multicolumn{2}{|c|}{2019} \\
\hline & $\%$ & (IC95\%) & $\%$ & (IC95\%) \\
\hline Brasil & 27,9 & $(27,1-28,8)$ & 28,5 & $(27,8-29,2)$ \\
\hline \multicolumn{5}{|l|}{ Sexo } \\
\hline Masculino & 27,0 & $(26,1-27,9)$ & 27,4 & $(26,6-28,2)$ \\
\hline Feminino & 28,8 & $(27,9-29,7)$ & 29,5 & $(28,8-30,3)$ \\
\hline \multicolumn{5}{|l|}{ Faixa etária } \\
\hline$<18$ & 23,1 & $(22,1-24,1)$ & $25,4^{a}$ & $(24,5-26,4)$ \\
\hline $18-29$ & 26,1 & $(25,1-27,1)$ & 25,3 & $(24,4-26,1)$ \\
\hline $30-59$ & 31,1 & $(30,2-32,1)$ & 30,9 & $(30,1-31,8)$ \\
\hline $60+$ & 30,8 & $(29,4-32,3)$ & 30,2 & $(29,2-31,3)$ \\
\hline \multicolumn{5}{|l|}{ Cor ou raça } \\
\hline Branca & 37,9 & $(36,6-39,2)$ & 38,8 & $(37,7-39,9)$ \\
\hline Preta & 21,6 & $(20,0-23,3)$ & 21,4 & $(20,3-22,6)$ \\
\hline Parda & 18,7 & $(18,0-19,4)$ & $20,1^{a}$ & $(19,5-20,7)$ \\
\hline Outra & 32,7 & $(28,5-37,1)$ & 30,6 & $(26,2-35,4)$ \\
\hline \multicolumn{5}{|l|}{ Nível de instrução mais elevado alcançado } \\
\hline $\begin{array}{l}\text { Fundamental incompleto ou equivalente (incluindo sem } \\
\text { instrução) }\end{array}$ & 16,4 & $(15,7-17,2)$ & 16,1 & $(15,5-16,7)$ \\
\hline Fundamental completo ou equivalente & 22,8 & $(21,7-23,9)$ & $20,8^{\mathrm{a}}$ & $(20,0-21,7)$ \\
\hline Médio completo ou equivalente & 37,4 & $(36,4-38,4)$ & $34,1^{\mathrm{a}}$ & $(33,3-34,9)$ \\
\hline Superior completo & 68,8 & $(67,2-70,4)$ & 67,6 & $(66,4-68,8)$ \\
\hline \multicolumn{5}{|l|}{ Faixa de rendimento domiciliar per capita } \\
\hline Até 1/4 salário mínimo & 5,0 & $(3,9-6,2)$ & $3,0^{\mathrm{a}}$ & $(2,6-3,5)$ \\
\hline Mais de $1 / 4$ até $1 / 2$ salário mínimo & 7,2 & $(6,5-8,0)$ & 7,5 & $(6,9-8,2)$ \\
\hline Mais de $1 / 2$ até 1 salário mínimo & 17,1 & $(16,2-18,1)$ & 17,7 & $(16,9-18,5)$ \\
\hline Mais de 1 até 2 salários mínimos & 34,8 & $(33,5-36,2)$ & 35,7 & $(34,7-36,8)$ \\
\hline Mais de 2 até 3 salários mínimos & 56,5 & $(54,3-58,6)$ & 54,8 & $(53,0-56,7)$ \\
\hline Mais de 3 até 5 salários mínimos & 67,5 & $(65,0-69,9)$ & $72,2^{\mathrm{a}}$ & $(70,3-73,9)$ \\
\hline Mais de 5 salários mínimos & 84,1 & $(82,2-85,9)$ & $88,0^{\mathrm{a}}$ & $(86,6-89,2)$ \\
\hline \multicolumn{5}{|l|}{ Condição em relação à força de trabalho } \\
\hline Pessoas na força de trabalho & 31,6 & $(30,6-32,5)$ & 31,7 & $(30,9-32,5)$ \\
\hline Pessoas fora da força de trabalho & 24,7 & $(23,7-25,7)$ & 24,5 & $(23,7-25,3)$ \\
\hline \multicolumn{5}{|l|}{ Condição de ocupação } \\
\hline Pessoas Ocupadas & 32,5 & $(31,5-33,5)$ & 33,3 & $(32,5-34,2)$ \\
\hline Pessoas desocupadas & 16,3 & $(14,6-18,2)$ & 15,0 & $(13,9-16,1)$ \\
\hline \multicolumn{5}{|l|}{ Situação em relação a formalidade do trabalho } \\
\hline Ocupado em emprego formal ${ }^{\star}$ & - & - & 47,4 & $(46,4-48,5)$ \\
\hline Ocupado sem carteira assinada & - & - & 19,1 & $(18,4-19,9)$ \\
\hline
\end{tabular}

Para as mulheres que declararam possuir plano de saúde, a cobertura de atendimento ao parto foi de $80,3 \%$, variando de $61,2 \%$ entre as menores de 18 anos de idade e $87,9 \%$ entre as mulheres com ensino superior completo (Tabela 2).

Em relação ao pagamento das mensalidades do plano de saúde, considerando os dados da PNS/2019, cerca de $47 \%$ dos titulares efetuam o pagamento diretamente a operadora do plano, sendo $72,7 \%$ entre aqueles ocupados sem carteira de trabalho assinada e $30,7 \%$ entre os que possuem emprego formal (Figura 2). A PNS/2019 permite diferenciar aqueles que possuem cobertura exclusivamente médica, aqueles que possuem cobertura médica e odontológica simultaneamente e os que possuem cobertura exclusi- 
Tabela 1. Percentual de pessoas com posse de plano de saúde médico ou odontológico e intervalo de confiança de 95\%, segundo características sociodemográficas. Brasil, 2013 e 2019.

\begin{tabular}{|c|c|c|c|c|}
\hline \multirow{2}{*}{ Variáveis } & \multicolumn{2}{|r|}{2013} & \multicolumn{2}{|c|}{2019} \\
\hline & $\%$ & (IC95\%) & $\%$ & (IC95\%) \\
\hline \multicolumn{5}{|l|}{ Tipo de situação censitária } \\
\hline Urbano & 31,7 & $(30,7-32,7)$ & 32,2 & $(31,3-33)$ \\
\hline Rural & 6,2 & $(5,3-7,3)$ & 7,0 & $(6,3-7,8)$ \\
\hline \multicolumn{5}{|l|}{ Tipo de área } \\
\hline Capital & 40,1 & $(38,9-41,3)$ & $42,4^{\mathrm{a}}$ & $(41,1-43,7)$ \\
\hline Resto da RM (Região Metropolitana, excluindo a capital) & 31,3 & $(29,6-33,1)$ & 32,6 & $(30,9-34,4)$ \\
\hline RIDE (excluindo a capital) & 14,0 & $(10,6-18,2)$ & 18,7 & $(12,7-26,7)$ \\
\hline $\begin{array}{l}\text { Resto da UF (Unidade da Federação, excluindo a região } \\
\text { metropolitana e RIDE) }\end{array}$ & 22,3 & $(21,1-23,5)$ & 21,8 & $(20,9-22,9)$ \\
\hline \multicolumn{5}{|l|}{ Região } \\
\hline Norte & 13,3 & $(12,3-14,5)$ & 14,7 & $(13,6-16,0)$ \\
\hline Nordeste & 15,5 & $(14,6-16,4)$ & 16,6 & $(15,8-17,4)$ \\
\hline Sudeste & 36,9 & $(35,2-38,6)$ & 37,5 & $(36,0-39,0)$ \\
\hline Sul & 32,8 & $(30,5-35,2)$ & 32,8 & $(31,3-34,3)$ \\
\hline Centro-Oeste & 30,3 & $(28,7-32,1)$ & 28,9 & $(27,2-30,7)$ \\
\hline
\end{tabular}

* Servidor público, trabalhador com carteira assinada, empregador ou militar. ${ }^{\text {a }}$ Diferença significativa entre as estimativas de 2013 e 2019, baseadas em um valor de p menor que 0,01 no teste t de Student para amostras independentes

Fonte: Pesquisa Nacional de Saúde (PNS), 2013; 2019.

vamente odontológica. A parcela de pessoas com cobertura exclusivamente odontológica é maior entre aqueles com piores condições socioeconômicas e os desocupados (Figura 3). Para o total da população brasileira, 26\% (IC95\%: 25,3\%$26,8 \%)$ possuem plano de saúde com cobertura médica e 2,5\% (IC95\%: 2,3\%-2,6\%) possuem cobertura exclusivamente odontológica (dados não apresentados na figura).

\section{Discussão}

O percentual de pessoas cobertas por plano de saúde se manteve estável neste período. Embora a tendência seja de crescimento deste percentual ao longo dos últimos anos, variando de 24,5\% em $1998^{13}$ para 28,5 em 2019, a variação entre 2013 e 2019 foi muito pequena e não foi significativa. Entretanto, houve variação em alguns estratos da população. A cobertura por plano de saúde diminuiu significativamente entre aqueles com rendimento domiciliar per capita de até um quarto do salário mínimo e indivíduos com ensino fundamental completo e médio completo. Já entre pessoas com melhor rendimento domiciliar per capita, acima de três salários mínimos e pessoas residentes em capitais, houve um aumento significativo desta cobertura.
Dados do Painel de Precificação dos Planos de Saúde da Agência Nacional de Saúde Suplementes (ANS), edição de julho de 2020, mostram que o valor médio comercial dos planos de saúde contratados pela modalidade coletivo empresarial, que agrega quase $70 \%$ dos usuários, teve um aumento de $27 \%$ entre 2016 e 2019, passando de 391 reais para 495 reais (http://www.ans.gov.br/aans/noticias-ans/ numeros-do-setor/5980-ans-disponibilizapainel-de-precificacao-em-novo-formato). Este aumento no valor médio comercial dos planos de saúde ocorreu também entre os planos de saúde contratados individualmente e por adesão coletiva. Neste mesmo período, o salário mínimo oficial passou de 880 reais em 2016 para 998 reais em 2019, representando um acréscimo de 13\%.

Segundo os dados da PNS/2013 e da PNS/2019, a proporção de pessoas desocupadas, ou seja, aquelas dentro da força de trabalho que estão desempregadas, passou de 5,8\% (IC95\%: 5,5\%-6,0\%) em 2013, para 9,1\% (IC95\%: 8,8\%$9,4 \%$ ) em 2019 (estimativas feita pelos autores). Tendo em vista que a maior parte dos planos de saúde são contratados via empregador, isso pode ter impacto na cobertura por plano de saúde.

Apesar das limitações dos indicadores apresentados nos parágrafos anteriores, estas informações Apesar das limitações dos indicadores 


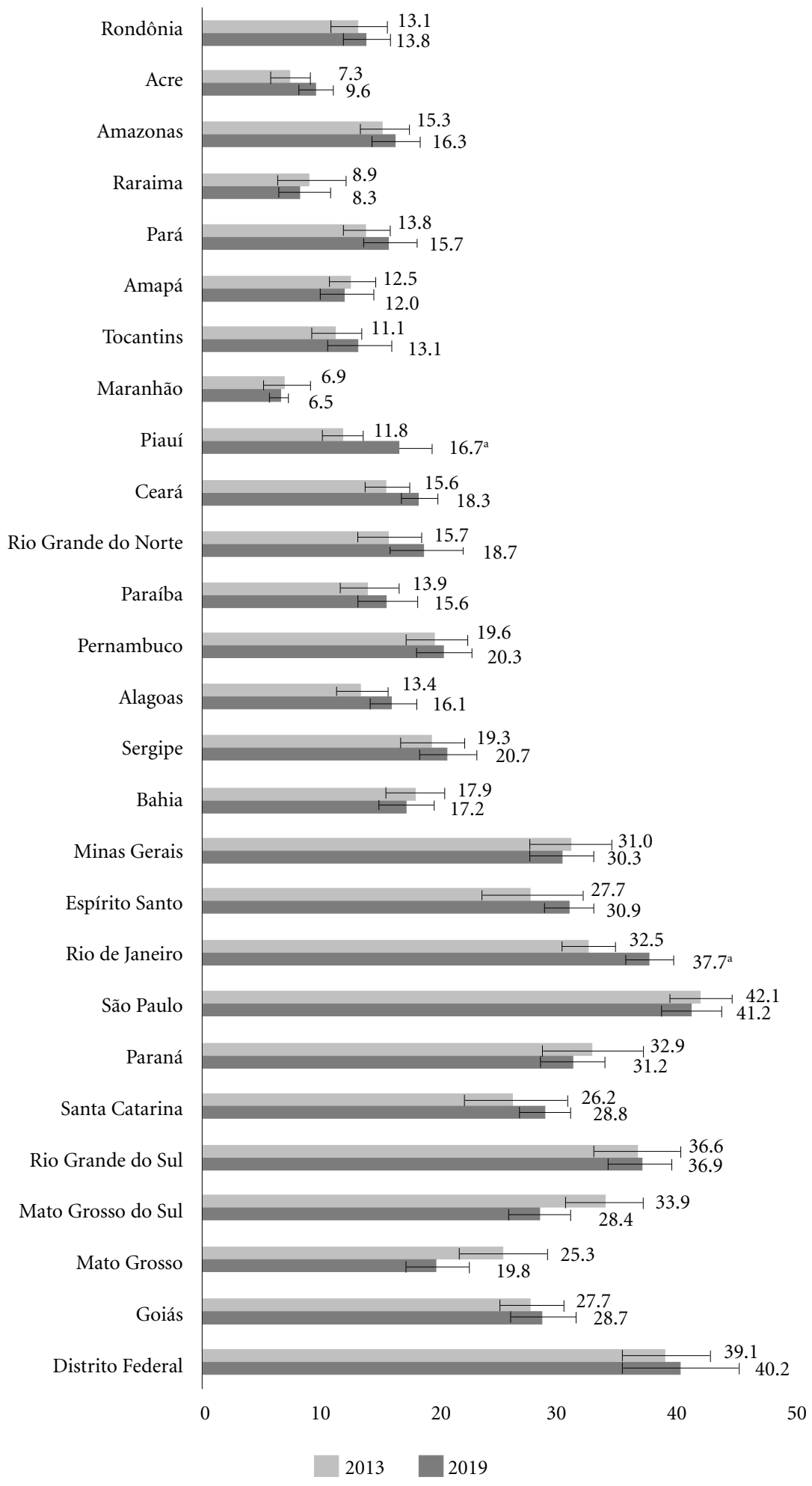

Figura 1. Cobertura de plano de saúde segundo Unidade da Federação. Brasil, 2013 e 2019.

a Diferença significativa entre as estimativas de 2013 e 2019, baseadas em um valor de p menor que 0,01 no teste t de Student para amostras independentes. 


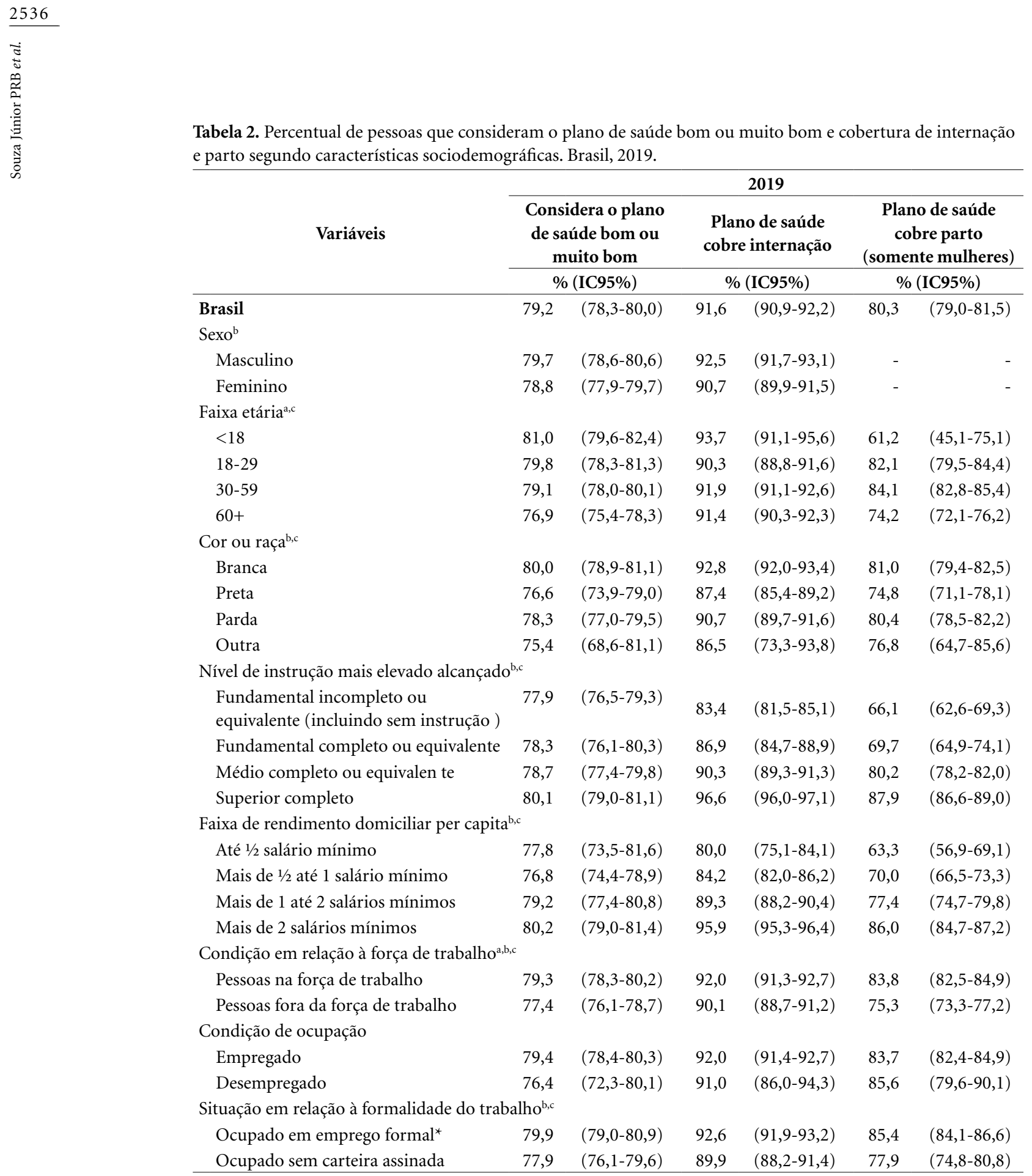

continua

produzem algumas hipóteses sobre a redução da cobertura em alguns estratos socioeconômicos e a manutenção do percentual de pessoas cobertas por plano de saúde no Brasil.

Outra questão importante que pode nortear este debate é a expansão da Estratégia de Saúde da Família (ESF), principalmente em municípios do interior e das regiões metropolitanas e em áreas onde residem populações com menor nível so- cioeconômico. Pinto e Giovanella ${ }^{14}$ estimam que a cobertura da ESF (inicialmente Programa de Saúde da Família) passou de 4,4\% em 1998 para $70 \%$ em 2017, destacando que nos municípios do interior do País, a cobertura chegou a 76,5\% enquanto nas capitais alcançou 45,5\%. Esta ampliação do acesso a serviços de saúde pode ter desestimulado as famílias com menor renda a contratar planos de saúde. 
Tabela 2. Percentual de pessoas que consideram o plano de saúde bom ou muito bom e cobertura de internação e parto segundo características sociodemográficas. Brasil, 2019.

\begin{tabular}{|c|c|c|c|c|c|c|}
\hline \multirow{3}{*}{ Variáveis } & \multicolumn{6}{|c|}{2019} \\
\hline & \multirow{2}{*}{\multicolumn{2}{|c|}{$\begin{array}{c}\begin{array}{c}\text { Considera o plano } \\
\text { de saúde bom ou } \\
\text { muito bom }\end{array} \\
\%(\mathrm{IC} 95 \%) \\
\end{array}$}} & \multicolumn{2}{|c|}{$\begin{array}{l}\text { Plano de saúde } \\
\text { cobre internação }\end{array}$} & \multicolumn{2}{|c|}{$\begin{array}{c}\text { Plano de saúde } \\
\text { cobre parto } \\
\text { (somente mulheres) }\end{array}$} \\
\hline & & & \multicolumn{2}{|c|}{$\%($ IC95\%) } & \multicolumn{2}{|c|}{$\%($ IC95\%) } \\
\hline \multicolumn{7}{|l|}{ Tipo de situação censitária ${ }^{\mathrm{b}, \mathrm{c}}$} \\
\hline Urbano & 79,2 & $(78,3-80,0)$ & 91,8 & $(91,1-92,4)$ & 80,5 & $(79,3-81,8)$ \\
\hline Rural & 80,0 & $(76,5-83,1)$ & 85,6 & $(82,3-88,3)$ & 70,2 & $(65,1-74,9)$ \\
\hline \multicolumn{7}{|l|}{ Tipo de área $a^{\mathrm{b}, \mathrm{c}}$} \\
\hline Capital & 79,2 & $(78,2-80,2)$ & 94,5 & $(93,9-95,1)$ & 82,3 & $(81,0-83,5)$ \\
\hline $\begin{array}{l}\text { Resto da RM (Região Metropolitana, } \\
\text { excluindo a capital) }\end{array}$ & 78,2 & $(76,2-80,0)$ & 89,1 & $(87,7-90,4)$ & 80,5 & $(78,1-82,8)$ \\
\hline RIDE (excluindo a capital) & 79,3 & $(64,6-89,0)$ & 87,5 & $(76,8-93,6)$ & 70,6 & $(54,9-82,7)$ \\
\hline $\begin{array}{l}\text { Resto da UF (Unidade da Federação, } \\
\text { excluindo a região metropolitana e } \\
\text { RIDE) }\end{array}$ & 79,6 & $(78,0-81,1)$ & 90,1 & $(88,8-91,3)$ & 78,4 & $(76,0-80,7)$ \\
\hline \multicolumn{7}{|l|}{ Região $^{\mathrm{a}, \mathrm{b}, \mathrm{c}}$} \\
\hline Norte & 77,1 & $(73,4-80,4)$ & 93,8 & $(92,2-95,2)$ & 82,6 & $(79,7-85,1)$ \\
\hline Nordeste & 73,8 & $(71,9-75,6)$ & 93,3 & $(92,0-94,5)$ & 81,4 & $(79,4-83,3)$ \\
\hline Sudeste & 79,4 & $(78,1-80,7)$ & 92,2 & $(91,2-93,0)$ & 81,4 & $(79,4-83,2)$ \\
\hline Sul & 82,4 & $(80,8-83,9)$ & 86,8 & $(85,2-88,3)$ & 72,8 & $(70,2-75,2)$ \\
\hline Centro-Oeste & 81,7 & $(79,9-83,4)$ & 93,1 & $(91,5-94,5)$ & 85,0 & $(82,4-87,2)$ \\
\hline
\end{tabular}

Ocupado em cargo formal ${ }^{\star}$

Ocupado sem carteira assinada

Desocupado

Fora da força de trabalho

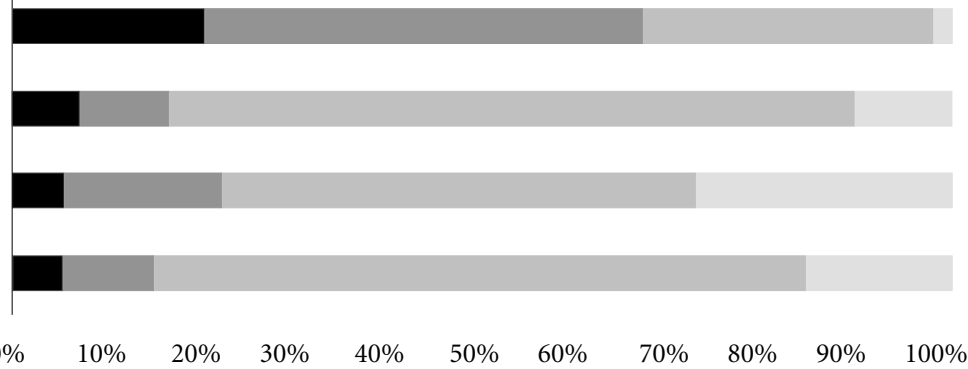

Somente o empregador

Parte o titular e parte o empregador

Somente o titular, diretamente ao plano

Outros**

Figura 2. Distribuição do responsável pelo pagamento da mensalidade do plano de saúde segundo a situação de ocupação e a força de trabalho. Brasil, 2019. * Servidor público, trabalhador com carteira assinada, empregador ou militar. ** Agregação das categorias "Somente outro
morador do domićlilio", "Pessoa não moradora do domicílio" e "Outro". 


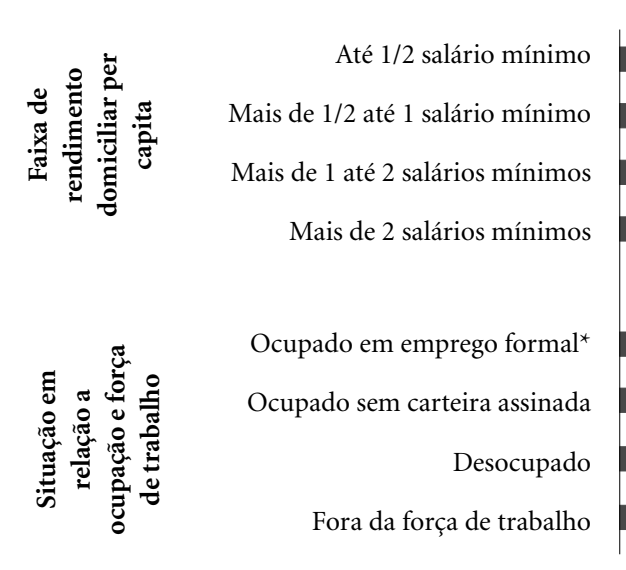

$\begin{array}{lllllllllllll}0 \% & 10 \% & 20 \% & 30 \% & 40 \% & 50 \% & 60 \% & 70 \% & 80 \% & 90 \% & 100 \%\end{array}$

Exclusivamente médico

Médico e odontológico

Exclusivamente dental

Figura 3. Distribuição do tipo de cobertura assistencial do plano de saúde segundo a faixa de rendimento domiciliar per capita e a situação em relação a ocupação e a força de trabalho. Brasil, 2019.

* Servidor público, trabalhador com carteira assinada, empregador ou militar.

Os resultados do presente estudo mostram que a cobertura de plano de saúde continua concentrada nos grandes centros urbanos, nas regiões Sudeste e Sul, entre aqueles com melhor nível socioeconômico e entre aqueles que possuem algum vínculo de trabalho formal, com carteira assinada, como empregadores ou servidores públicos e militares. Outros estudos também verificaram estas características entre as pessoas com posse de plano de saúde ent,15-19. $^{13}$.

Entre as pessoas que residem em municípios das Regiões Metropolitanas (excluindo as capitais), o percentual de posse de plano de saúde médico ou odontológico foi de $32,6 \%$, porém, nas Regiões Integradas de Desenvolvimento (RIDE) (excluindo as capitais), a cobertura é de 18,7\%, sendo menor que a cobertura nos municípios do interior (restante dos municípios das UF com exceção das capitais, RM e RIDE). Apesar destas áreas serem semelhantes às RM, exceto pelo fato de incorporarem municípios de diferentes UF, elas possuem uma estrutura mais precária e uma população residente com piores condições socioeconômicas, tendo, segundo dados da própria PNS/2019, quase 70\% do conjunto de sua população com 14 anos ou mais com renda per capita de até um salário mínimo. Estes resultados mostram a necessidade dos serviços públicos de saú- de serem suficientes para atender as demandas da população destas RIDE. O estudo de Fernandes et al. $^{20}$, realizado no ano 2012 com a população do Entorno Norte, uma sub-região da RIDE do Distrito Federal (RIDE-DF) discorre que 38\% de todas a instituições de saúde locais são unidades privadas e que isso pode ser um indicativo da falta de serviços públicos de saúde nesta região. Da mesma forma, Rocha ${ }^{21}$ analisando dados dos anos 2010 e 2011 do Entorno Sul da RIDE-DF, afirma que apenas 20,4\% dos domicílios da região eram cadastrados na Estratégia de Saúde da Família (ESF) entre 2010 e 2011 e que este percentual era bem abaixo da média de cobertura nacional da ESF da época (54\%).

Em relação ao maior percentual de posse de plano de saúde médico ou odontológico entre os trabalhadores mais formalizados, Machado et al. ${ }^{18}$ também encontraram uma relação positiva entre estes, e seus rendimentos, com a cobertura de plano de saúde, e destacam que: "Tal relação sugere o papel que as grandes empresas, sejam elas públicas ou privadas, detêm na criação de um mercado de trabalho mais 'protegido', contribuindo colateralmente para fomentar o mercado de planos coletivos privados"18(p.766). Os autores ressaltam que estas diferenças entre os postos de trabalho formal e informal, como a oferta de pla- 
no de saúde para os trabalhadores, promove ainda mais a segmentação e dificulta a mobilidade dos trabalhadores entre estes grupos, pois aqueles trabalhadores com maior acesso ao plano de saúde têm melhores condições de acumular capital humano.

Outra diferença relevante em relação aos trabalhadores com emprego formal e aqueles com trabalho informal é que, mesmo quando ambos são benificiários de plano de saúde, os custos sobre o mesmo são bem diferentes. Entre os trabalhadores formalizados, somente 30,7\% relatou que o pagamento da mensalidade é feito diretamente à operadora, enquanto $72,7 \%$ dos trabalhadores informais pagam os planos desta maneira. Ou seja, os custos do plano de saúde são muito maiores para os trabalhadores informais, comprometendo uma parcela maior do salário. Uma pesquisa com dados da Pesquisa de Orçamento Familiar (POF) de 2002-2003, inquérito populacional realizado pelo IBGE, analisou o gasto catastrófico em saúde, ou seja, gasto com saúde maior ou igual a $40 \%$ da capacidade de pagamento das famílias. Os autores, baseados nos resultados, sugerem que as famílias com seguro saúde estão, na maioria dos casos, expostas a um risco maior de gastos catastróficos com saúde do que aquelas que não têm seguro ${ }^{22}$.

Podemos ressaltar também, que dentre as pessoas desocupadas, ou seja, aquelas que estão procurando emprego, apenas 15\% (em 2019) relatou possuir plano de saúde. Embora toda a população brasileira esteja coberta pelo SUS, aqueles que possuem acesso aos serviços privados de saúde através do plano, possuem cobertura duplicada e maior facilidade de acesso aos serviços, pois podem optar por serviços de ambos os setores ${ }^{2,23}$. De fato, a oferta de leitos privados é bem maior que de leitos públicos (SUS), sendo estimada por Santos et al. ${ }^{2}$ em 2,9 leitos/ mil habitantes, contra 1,8 leitos por mil habitantes no SUS, sendo $60 \%$ maior a oferta de leitos por habitante no setor privado em 2005. Chama a atenção também, a diferença na oferta de equipamentos de média e alta complexidade, tendo a rede privada cerca de 4 vezes mais tomógrafos computadorizados por habitante e 5 vezes mais mamógrafos e ultrassonografias por habitante que aqueles disponíveis na rede pública². Um estudo realizado com dados do Sistema de Vigilância de Fatores de Risco e Proteção para Doenças Crônicas por Inquérito Telefônico (Vigitel) do ano 2015 mostrou que, para o conjunto das capitais brasileiras, a realização de exames de mamografia para mulheres entre 50 e 69 anos e do exame de Papanicolau, para mulheres de 25 a 64 anos, foi maior na população coberta por plano de saúde, mesmo após estratificação pelo nível socioeconômico ${ }^{17}$. De fato, as taxas de utilização de serviços de saúde são maiores entre as pessoas cobertas por plano de saúde ${ }^{24}$.

Cerca de $92 \%$ dos planos de saúde médico cobrem internação, sendo pouco mais de $8 \%$, com cobertura ambulatorial somente. Olhando os dados disponíveis no site da Agência Nacional de Saúde Suplementar (http://www.ans.gov.br/ anstabnet/cgi-bin/dh?dados/tabnet_br.def), para dezembro de 2019, cerca de $4 \%$ dos beneficiários de plano de saúde possuem planos com cobertura somente ambulatorial. Nossos resultados mostram um gradiente da cobertura de internação de acordo com o nível de instrução e as classes de renda per capita, sendo maior a cobertura quanto maior a escolaridade e a renda. Dentre as pessoas com renda per capita até um quarto do salário mínimo e que relataram possuir plano, cerca de $20 \%$ não possuem cobertura para internação, reforçando a importância do SUS para esta população. Analisando dados da Pesquisa Nacional por amostra de Domicílios (PNAD) de 2008, Porto et al. ${ }^{24}$ relatam que "nada menos que $9 \%$ das cirurgias realizadas pelo SUS foram usadas por essas pessoas que têm dupla cobertura na atenção à saúde"24(p.3804), referindo-se às pessoas cobertas por plano de saúde.

Entre as mulheres que possuem plano de saúde, quase $20 \%$ delas não possuem cobertura para o parto, necessitando recorrer ao SUS ou ao pagamento direto para a realização do mesmo. Este percentual também varia de acordo com o nível socioeconômico, sendo apenas 63,3\% para aquelas com renda familiar per capita de até um quarto do salário mínimo, chegando a $86 \%$ entre aquela com renda per capita maior que dois salários-mínimos. Estes resultados mostram a importância do SUS e das políticas públicas voltadas para a saúde da mulher e a assistência ao parto, pois apenas $11,7 \%$ das mulheres brasileiras com idade entre 15 e 44 anos possuem cobertura para o parto através do plano de saúde. Segundo Porto et al. ${ }^{24}, 97,7 \%$ dos partos normais e 58,4\% dos partos cesáreos, no ano de 2008, foram financiados pelo SUS.

Por fim, este estudo mostrou que a proporção de planos exclusivamente odontológicos é maior entre aqueles com menor nível socioeconômico, sugerindo que esta população deve ter alguma dificuldade de aceso a atendimentos odontológicos no SUS, comprometendo parte de seus rendimentos a este tipo de plano de saúde. Este percentual 
também foi maior estre as pessoas desocupadas. Estudo realizado em 2005, em quatro municípios do estado do Rio de Janeiro, mostrou maior frequência de visita odontológica regular entre os moradores de domicílios de baixa renda não cobertos pelo Programa de Saúde da Família (PSF) quando comparados àqueles moradores de domicílios de baixa renda cobertos pelo PSF, mas sem implantação de Equipes de Saúde Bucal (ESB) ${ }^{25}$.

A utilização dos serviços odontológicos no Brasil segue um padrão ditado pelas desigualdades sociais, sendo a maior proporção de uso entre indivíduos de maior renda e à medida que a idade aumenta, menor é a proporção de uso de serviços odontológicos ${ }^{26,27}$ e ainda, sendo $50 \%$ das consultas odontológicas realizadas com pagamento direto ${ }^{24,28}$. Entretanto, o número de beneficiários de planos exclusivamente odontológicos vem crescendo no Brasil ${ }^{29}$, em decorrência ao alto custo destes serviços.

Dentre as limitações do presente estudo, enfatiza-se que os dados utilizados da PNS, referentes a todos os moradores do domicílio, foram referidos por um dos residentes (proxy), o que pode gerar erros nas respostas e a interpretações equivocadas da pergunta ou desconhecimento, por parte do(a) respondente, de detalhes sobre o plano de saúde. Vale ressaltar que o IBGE utiliza neste módulo da pesquisa uma metodologia de coleta que permite que outra pessoa moradora do domicílio responda o questionário pelas pessoas que estão ausentes no momento da visita do entrevistador.

Para concluir, este estudo apresentou a cobertura de plano de saúde segundo diversas características dos respondentes, identificando que não houve um aumento relevante no percentual de pessoas cobertas e que as desigualdades nesta cobertura persistem. Estes resultados podem auxiliar os gestores de saúde no planejamento da assistência à saúde no SUS e reforça a importância do Sistema Único de Saúde na redução das desigualdades e na oferta de serviços públicos para a população de baixa renda, bem como, aquelas residentes em municípios menores ou menos desenvolvidos e em áreas rurais. Foi possível verificar também, que parte da população coberta por planos de saúde não possui cobertura para internação e/ou parto, sugerindo que esta população utiliza o SUS quando precisa destes serviços.

\section{Colaboradores}

PRB Souza Júnior foi responsável pela concepção e delineamento do artigo, análise e interpretação dos dados e redação do artigo. CL Szwarcwald, GN Damacena, SR Stopa e MM Oliveira contribuíram na interpretação dos dados, redação do artigo e revisão crítica. WS Almeida, LMV Sardinha, MLFP Vieira e EM Macário contribuíram na interpretação dos dados e revisão crítica do artigo.

\section{Financiamento}

Esta pesquisa não recebeu financiamento específico de nenhuma agência de fomento nos setores público, comercial ou sem fins lucrativos. Este artigo apresenta uma pesquisa independente. As opiniões expressas nesta publicação são dos autores e não necessariamente reconhecidas pelas instituições. 


\section{Referências}

1. Brasil. Constituição da República Federativa do Brasil de 1988. Diário Oficial da União 1988; 5 out.

2. Santos IS, Ugá MAD, Porto SM. O mix público-privado no Sistema de Saúde Brasileiro: financiamento, oferta e utilização de serviços de saúde. Cien Saude Colet 2008; 13(5):1431-1440.

3. Bonfada D. Gasto com a internação de idosos em unidades de terapia intensiva [tese]. Natal: Universidade Federal do Rio Grande do Norte; 2015.

4. Bahia L. As contradições entre o SUS universal e as transferências de recursos públicos para os planos e seguros privados de saúde. Cien Saude Colet 2008; 13(5):1385-1397.

5. Brasil. Lei $n^{\circ} 9.656$, de 3 de junho de 1998. Regulamenta a Saúde Suplementar no Brasil e dá outras providências. Diário Oficial da União 1998; 2 jun.

6. Brasil. Lei $\mathrm{n}^{\circ}$ 9.961, de 28 de janeiro de 2000. Cria a Agência Nacional de Saúde Suplementar - ANS e dá outras providências. Diário Oficial da União 2000; 28 jan.

7. Szwarcwald CL, Malta DC, Pereira CA, Vieira MLFP, Conde WL, Souza-Júnior PRB, Damacena GN, Azevedo LO, Azevedo e Silva G, Theme-Filha MM, Lopes CS, Romero DE, Almeida WS, Monteiro CA. Pesquisa Nacional de Saúde no Brasil: concepção e metodologia de aplicação. Cien Saude Colet 2014; 19(2):333342.

8. Souza-Júnior PRB, Freitas MPS, Antonaci GA, Szwarcwald CL. Desenho da amostra da Pesquisa Nacional de Saúde 2013. Epidemiol Serv Saude 2015; 24(2):207216.

9. Instituto Brasileiro de Geografia e Estatística (IBGE) Coordenação de Trabalho e Rendimento. Pesquisa nacional de saúde: 2019: informações sobre domicílios, acesso e utilização dos serviços de saúde: Brasil, grandes regiões e unidades da federação. Rio de Janeiro: IBGE; 2020.

10. IBM SPSS Statistics for Windows [programa de computador]. Versão 21.0. Armonk (NY): IBM Corp; 2012.

11. Lee S, Davis WW, Nguyen HA, McNeel TS, Brick JM, Flores-Cervantes I. Examining trends and averages using combined cross-sectional survey data from multiple years. CHIS Methodology Paper 2007; 1-26.

12. Nelson DE, Powell-Griner E, Town M, Kovar MG. A comparison of national estimates from the National Health Interview Survey and the Behavioral Risk Factor Surveillance System. Am J Public Health 2003. 93(8):1335-1341.

13. Viacava F, Bellido JG. Condições de saúde, acesso a serviços e fontes de pagamento, segundo inquéritos domiciliares. Cien Saude Colet 2016; 21(2):351-370.

14. Pinto LF, Giovanella L. Do Programa à Estratégia Saúde da Família: expansão do acesso e redução das internações por condições sensíveis à atenção básica (ICSAB). Cien Saude Colet 2018; 23(6):1903-1914.

15. Malta DC, Moura EC, Oliveira M, Santos FP. Usuários de planos de saúde: morbidade referida e uso de exames preventivos, por inquérito telefônico, Brasil, 2008. Cad Saude Publica 2011; 27(1):57-66.

16. Malta DC, Stopa SR, Pereira CA, Szwarcwald CL, Oliveira M, Reis AC. Cobertura de Planos de Saúde na população brasileira, segundo a Pesquisa Nacional de Saúde, 2013. Cien Saude Colet 2017; 22(1):179-190.
17. Malta DC, Bernal RTI, Vieira-Neto E, Curci KA, Pasinato MTM, Lisbôa RM, Cachapuz RF, Coelho KSC, Santos FP, Freitas MIF. Doenças Crônicas Não Transmissíveis e fatores de risco e proteção em adultos com ou sem plano de saúde. Cien Saude Colet 2020; 25(8):2973-2983.

18. Machado AF, Andrade MV, Maia AC. A relação entre estrutura ocupacional e acesso a plano de saúde no Brasil: uma análise para 1998 e 2003. Cad Saude Publica 2012; 28(4):758-768.

19. Fontenelle LF, Camargo MBJ, Bertoldi AD, Gonçalves H, Maciel ELN, Barros AJD. Cobertura por plano de saúde ou cartão de desconto: inquérito domiciliar na área de abrangência da Estratégia Saúde da Família. Cad Saude Publica 2017; 33(10):e00141515.

20. Fernandes SES, Urdaneta M, Ferreira Noronha E, Merchan-Hamann E. Condições de saúde, uso de serviços e percepções da vizinhança. Inquérito no entorno de Brasília. Com Cien Saúde 2020; 31(2):43-54.

21. Rocha PRS. Situação de saúde de adultos residentes nos municípios do entorno sul da Região Integrada de Desenvolvimento do Distrito Federal e Entorno (RIDEDF): inquérito de base populacional, 2010-2011 [dissertação]. Brasília: Universidade de Brasília; 2012.

22. Barros AJD, Bastos JL, Dâmaso AH. Gastos catastróficos com saúde no Brasil: seguro saúde privado não parece ser a solução. Cad Saude Publica 2011; 27(Supl. 2):s254-s262.

23. Porto SM, Santos IS, Ugá MAD. A utilização de serviços de saúde por sistema de financiamento. Cien Saude Colet 2006; 11(4):895-910.

24. Porto SM, Ugá A, Moreira R. Uma análise da utilização de serviços de saúde por sistema de financiamento. Cien Saude Colet 2011; 16(9):3795-3807.

25. Mendonça HLC, Szwarcwald CL, Damacena GN. Autoavaliação de saúde bucal: resultados da Pesquisa Mundial de Saúde - Atenção Básica em quatro municípios do Estado do Rio de Janeiro, Brasil, 2005. Cad Saude Publica 2012; 28(10):1927-1938.

26. Lima-Costa MF, Matos DL, Camarano AA. Evolução das desigualdades sociais em saúde entre idosos e adultos brasileiros: um estudo baseado na Pesquisa Nacional por Amostra de Domicílios (PNAD 1998, 2003). Cien Saude Colet 2006; 11(4):941-950.

27. Celeste RK, Nadanovsky P, Fritzell J. Trends in socioeconomic disparities in the utilization of dental care in Brazil and Sweden. Scand J Public Health 2011; 39(6):640-648.

28. Bastos GA, Duca GF, Hallal PC, Santos IS. Utilização de serviços médicos no sistema público de saúde no Sul do Brasil. Rev Saude Publica 2011; 45(3):475-484.

29. Pietrobon L, Silva CM, Batista LRV, Caetano JC. Planos de assistência à saúde: interfaces entre o público e o privado no setor odontológico. Cien Saude Colet 2008; 13(5):1589-1599.

Artigo apresentado em 11/11/2020

Aprovado em 02/12/2020

Versão final apresentada em 04/12/2020

Editores-chefes: Maria Cecília de Souza Minayo, Romeu Gomes, Antônio Augusto Moura da Silva 
